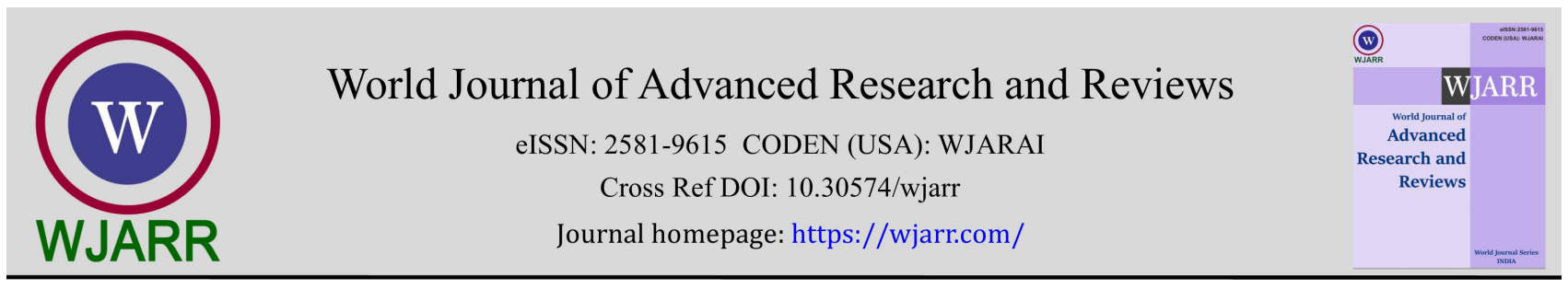

(RESEARCH ARTiCLE)

\title{
Big bang maps and color charge force
}

\author{
Gudrun Kalmbach H.E. *
}

MINT, PF 1533, D-86818 Bad Woerishofen, Germany.

World Journal of Advanced Research and Reviews, 2021, 12(02), 164-172

Publication history: Received on 10 March 2021; revised on 05 November 2021; accepted on 07 November 2021

Article DOI: https://doi.org/10.30574/wjarr.2021.12.2.0095

\begin{abstract}
The Planck and other natural numbers are used for units of forces. They arise also as weights of Gleason operators, defined by 3-dimensional spin-like base triples GF and their weigths. The spin lengths are the spin GF weights for instance. The measuring GF operator triples arise by projective duality from 1-dimensional force vectors in projective to $\mathrm{R}^{5}$ extended Hilbert space $\mathrm{H}^{4}$. Color charges are set as a separate force, using a G-compass (figure 2). For the universes evolution after a big bang several maps are introduced, mostly belonging to the gravity field quantum $r g b$-graviton. It presents the neutral color charge of nucleons. Orthogonal projections of $\mathrm{H}^{4}$, also in spiralic and angular form, central or stereographic projective maps belong to them. They project also the $S^{3}$ factor of the strong interation geometry $S^{3} \mathrm{xS}^{5}$ down to the SU(2) geometry $S^{3}$ of the Hopf map. Fiber bundle maps are added also to $\mathrm{S}^{5}$ with the same fiber $\mathrm{S}^{1}$ to the base space $\mathrm{CP}^{2}$ for nucleons and atomic kernels. In octonian coordinates, listed by indices, 01234567 , there are three projections from the energy space 123456 of SI to complex quaternionic $2 \times 2$ matrix presentations of spacetime 1234 , of $\mathrm{CP}^{2}$ as 3456 and of GR with mass and rgb-gravitons 1256 . GR and $\mathrm{CP}^{2}$ are projected into 1234 as the universes spacetime, observable as bubbles for atoms and matter 3456 and GR potentials and actions about and for mass carrying systems 1256.
\end{abstract}

Keywords: Color charge force; GF operator; Big bang; Planck numbers

\section{Planck Numbers and GF Operators}

In the list of Planck numbers (see [6]) is no constant mentioned for color charges. There are two experimentally determined constants for the strong interactions potential, but in this article they are not associated with color charge. In the list below, the octonian coordinate is added to which a force belongs as eigenvector of a symmtery. The coordinates are listed by their indices. Natural constants are for Planck numbers:

- $\gamma \mathrm{G}$ gravitational constant, occuring in the Schwarzschild radius Rs of a mass system. If Rs belongs to a black hole $Q$, the inversion of universes matter $P$ of radius $r$ when absorbed by $Q$ is using the mathematical inversion at a circle (figure 1) as radius inversion $r^{\prime} r=R s^{2}$. $P$ energy is stored in $Q$ having radius $r^{\prime}$.

If interpreted projective, it uses in a projective plane $\mathrm{P}^{2}$ the duality pole-polar, where from the point $\mathrm{P}$ a tangent is drawn to the circle and on the polar for the point, the interesection with the horizontal $\mathrm{x}$-axis is marked as image point $\mathrm{P}^{\prime}$. This belongs to the mass 5 as potential force of gravity $E($ pot $)$ and the complex eigenvector $\left(-p_{2}, 1\right)$ of the 2x2-matrix G with first row (1 -1), second row (1 0), as color charge is added c( $r$ ) turquoise. A second eigenvector (1.1) is for mass belonging to the id identity $2 \times 2$-matrix.

\footnotetext{
* Corresponding author: Gudrun Kalmbach HE

MINT, PF 1533, D-86818 Bad Woerishofen, Germany.

Copyright (C) 2021 Author(s) retain the copyright of this article. This article is published under the terms of the Creative Commons Attribution Liscense 4.0 .
} 
- $\mathrm{h}$ Planck constant; in the equation $\mathrm{E}=\mathrm{hf}, \Delta \mathrm{t}$ for a time interval as circulation time of $\mathrm{P}$ on a circle $\mathrm{C}$ of radius 1 is inverted (see figure 1 for the map exp) to $\mathrm{f}=1 / \Delta \mathrm{t}=\mathrm{n}, \mathrm{n}$ a natural winding number. Energy can only be stored in full windings $n$, computed by the complex residual contour integration $c \int \mathrm{dz} / \mathrm{z}$. The electromagnetic interaction EMI symmetry $U(1)$ is for $C$ and its universal covering $R$ is for the map $t \rightarrow \exp (i t)$. This belongs to frequency 6 as kinetic energy and momentum E(kin) as force and the 2x2-matrix $\alpha$ with first row $(01)$, second row ( -11$)$ with eigenvector $\left(1,-p_{1}\right)$ for momentum $\mathrm{p}=\mathrm{mv}$, as color charge is added $\mathrm{b}$ blue.

- $\mathrm{k}$ (Kelvin) Boltzmann constant; this belongs to the sign change of the complex polar coordinate 2 as $+\varphi \rightarrow-\varphi$, as exponential map $\exp (\mathrm{i} \varphi) \rightarrow \exp (-\mathrm{i} \varphi)$ reversing the rotational orientation on $\mathrm{C}$ from mathematical positive $+\varphi$ to clockwise $-\varphi$. It is also for reversing speeds in the Lorentz transformations from $+v \rightarrow-v$ between a system $Q$ observing with special relativistic speed $+v$ a measured sysem $P$ not in gravitational interaction with $Q$, and reversely $\mathrm{P}$ as observer measures $\mathrm{Q}$ with speed $-\mathrm{v}$. This has the time reversal operator $\mathrm{T}:+\Delta \mathrm{t} \rightarrow-\Delta \mathrm{t}$ of physics associated. It applies to the Euclidean metric for spacetime coordinates 1234, xyzt, available for its Hilbert space metric
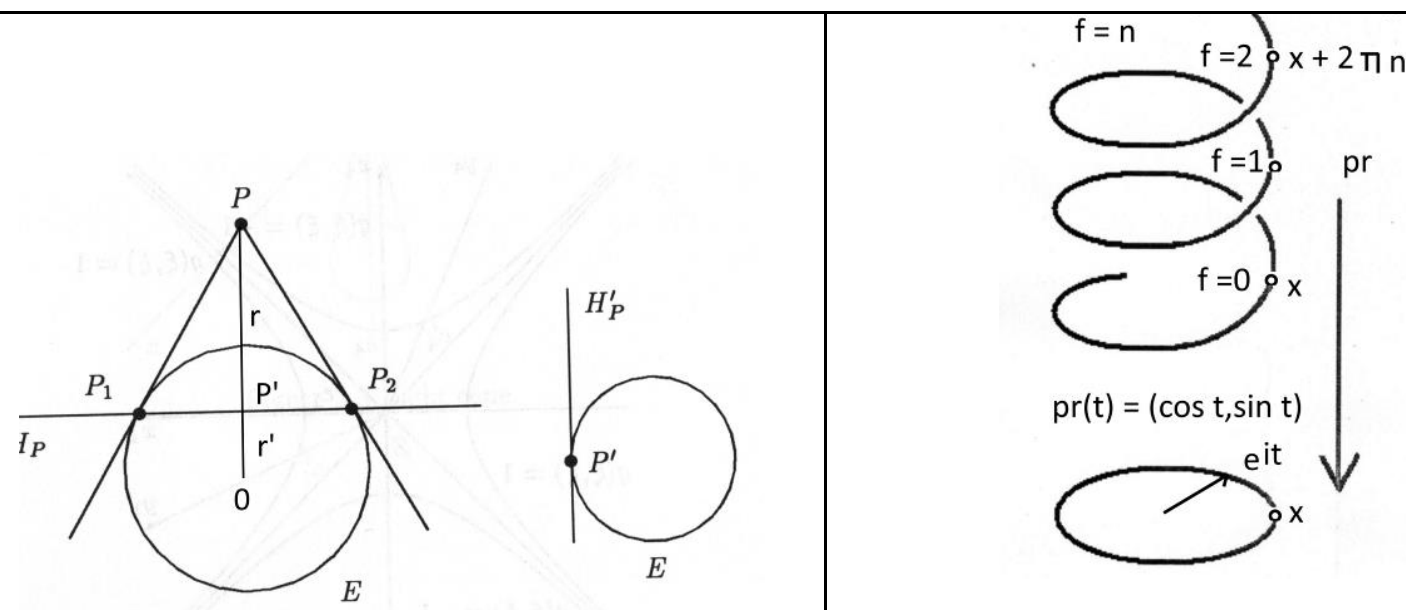

pole $\mathrm{P}$, polar $\mathrm{P}_{1} \mathrm{P}_{2}$, pole $\mathrm{P}^{\prime}$ incident with polar $\mathrm{H}_{\mathrm{p}}^{\prime}$ and quadric $E$ generated by these points for a correlation

Figure 1 Inversion at a circle, universal covering R as helix on a cylinder for wave expansions $\exp ($ it) in time

$<\mathrm{u}, \mathrm{u}>$ in $\langle\mathrm{uT}, \mathrm{u}\rangle=<(\mathrm{x}, \mathrm{y}, \mathrm{z},-\mathrm{ct}),(\mathrm{x}, \mathrm{y}, \mathrm{z}, \mathrm{ct})>=\mathrm{r}^{2}-\mathrm{c}^{2} \mathrm{t}^{2}, \mathrm{r}$ radius. Minkowski metric is generated. This belongs to heat 2 as temperature $\mathrm{E}$ (heat) as force and the $2 \mathrm{x} 2$-matrix $\alpha \sigma_{1}$ with first row (10), second row (1 - 1 ) with eigenvector $(0,1)$ or $(0, i)$, as color charge is added g green. As second Pauli matrix $\sigma_{2}$ for 2 , has additional an eigenvector $(i, i)$.

- c speed of light; EMI as force $\psi=\exp (\mathrm{i}(\omega t+\mathrm{xk}))$ has the coordinate 7 and no color charge attached. This is replaced by the exp function in figure 1 with the helix expansion of the waves $\psi$ frequency on a cylinder. $\Psi$ travels on its world line in time with speed c. An eigenvector $\left(-p_{1}, 1\right)$ for $U(1)$ is set by the octonian coordinate $e_{0}$ as G-compass needle which turns in powers of $G$ with the discrete sixth roots of unity. $G$ is of order 6 . The octonian subspace is 07 , a plane for the G-compass (figure 2).

Beside these big bang basic Planck time constants which define $\gamma \mathrm{G}$ mass $\left(\mathrm{m}=\mathrm{E} / \mathrm{c}^{2}\right)$ and length $\mathrm{l}$, $\mathrm{h}$ time $(\mathrm{t}=\mathrm{l} / \mathrm{c}$, as measure second $\mathrm{s}$ ) and energy ( $\mathrm{E}$, as measure $\mathrm{Hz}$ or $\mathrm{J})$, k heat $(\mathrm{T}=\mathrm{E} / \mathrm{k})$, additional density by $\rho=\mathrm{c}^{5} / \mathrm{h}(\gamma \mathrm{G})^{2}(\mathrm{as}$ measure mass $\mathrm{m}$ per volume $\mathrm{m} / \mathrm{l}^{3}$ ); c EMI waves, are listed in physics for computations, - the Schwarzschild radius Rs is set equal to a (de Broglie with $\lambda p=h$ ) wave length $\lambda=r=R s=2 m(\gamma G) / c^{2}, r$ radius. Mass mo is renormed when Higgs as field or boson sets mass $\mathrm{m}$ for wave packages like nucleons or atomic kernels, atoms.

The other forces, electromagnetic EM and weak interaction WI as EM or neutral charge contribute more natural constants for EM e, neutral $v$ forces, magnetic strength $\Phi$ and momentum $p=m v$ as E(kin) force. Spin as a 3 dimensional orthogonal base triple $s=\left(s_{x}, S_{y}, s_{z}\right)$ in space is a weak WI measuring apparatus, having a quasiparticle spinor associated. Spin length is quantized through natural winding numbers $n=1,2,3, \ldots$ about a circular cone (figure 1). The rotational force $\mathrm{E}(\mathrm{rot})$ is on $3 \mathrm{~s}$ and extends projective: in 5 real dimensions projective duality allows that correlations associate with 0 -dimensional points a hyperplane, with 1-dimensional force vectors a measuring space-like base triple. Spin 123 is the first example for WI with symmetry SU(2). Octonians have seven of this kind, 
145 for EM, 167 for EMI, 246 for E(heat) temperature, 257 for mass, Higgs and barycenters, 347 for E(rot), 356 for the strong interaction SI rotor. A SI base triple is 126 for $r g b$-gravitons, belonging to the first three GellMann 3x3matrices of the SU(3) symmetry. The conic color charge whirls red $r$, green g, blue b are in superposition.

The symmetries matrices have eigenvectors for the forces, as listed in the octonian coordinate notation. The e $0 \mathrm{G}$ compass (figure 2 left) needle has an $\left(-\mathrm{p}_{2}, 1\right)$ eigenvector of $\mathrm{G}$ representation for the color charge energy. This is repeated for other cross ratio energies with color charges attached. The id matrix for scalings and the EM charge 1 has the force eigenvector $(1,0), 2$ heat $(0,1)$ or $(0, i), 4$ magnetic energy $(i, 0), 5$ mass $(1,1), 6$ frequency $(1,-p 1)$. In figure 2 right are shown 1 red as vector E(pot) 5, 2 green as vector E(rot) 3 and 6 blue E(kin) as vector for the SI rotors 6-cycle dynamics as observables.

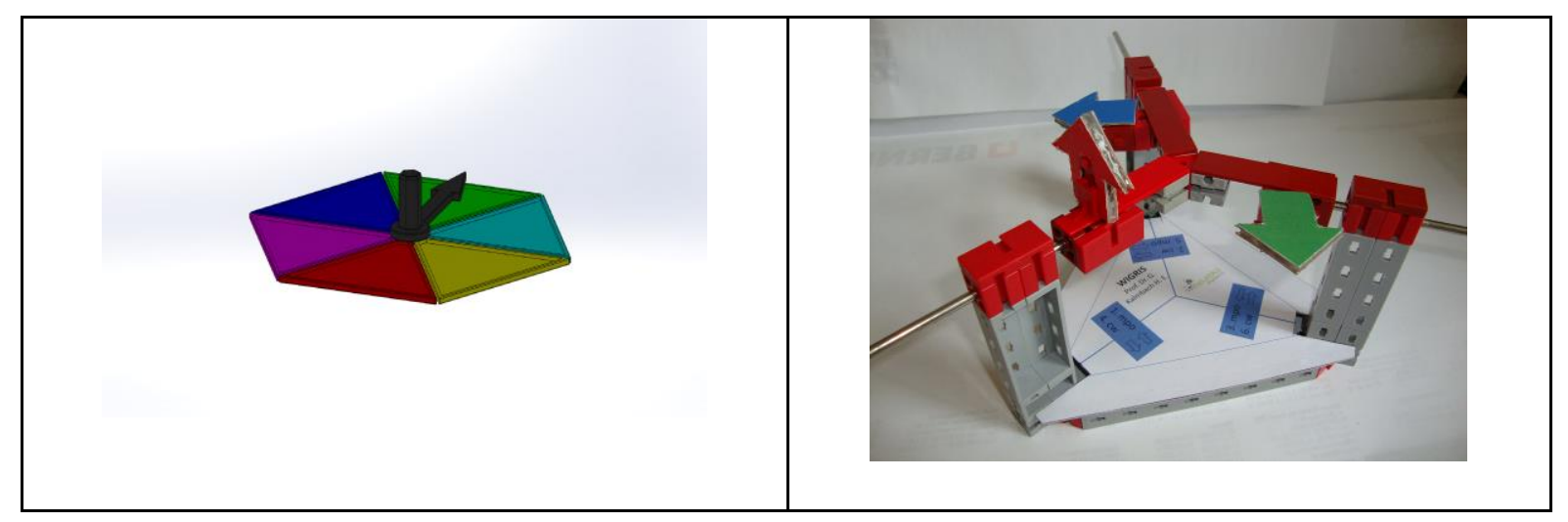

Figure 2 G-compass, SI rotor [7]

Planck numbers are extended: Physics has natural coupling constants for SI $\alpha_{\mathrm{s}}$, for EM $\alpha$ Sommerfeld fine structure constant, for WI $\alpha_{\mathrm{W}}$, for gravity GR $\alpha_{\mathrm{g}}$. There is missing an independent treatment for color charges. They are used as quantum number for quarks in the neutral rgb symmetry (a GF triple) form for baryons and as source for SI. The real part rgb is Heisenberg uncertainty complex extended where the imaginary parts $c(x), x=r, g, b$ turquoise, magenta, yellow are undetermined in measurements. No GF base triples are named in physics for EM (here 145), SI (here 356), WI (here 123), EMI (here 167). For GR rgb is not a symmetry as shown in the next section, E(rot) (347), E(kin) (356), E(heat) (246), E(pot) (257 mass) are missing in internet lists, also natural constants for them. Available are for instance three natural constants which use mol (Avogadro constant, Faraday, universal gas constants) and the Stefan Boltzmann constant, also for the complex G-compass color charge force.

\section{Color Charge Force}

As independent force is introduced the G-compass (see [3]. It uses complex numbers C in form of the Riemannian sphere $S^{2}$ with a stereographic point $\infty$ added. The stereographic map onto $C$ as tangent plane in the south pole with $\infty$ as north pole is the first map used by $r g b$-gravitons. More general they use central projections (figure 3).

The $r g b$-graviton symmetry is given by the six complex cross ratios as invariants of Moebius transformations MT, the $S^{2}$ symmetry. Permuted are 0,1 (or -1$), \infty$ in $(z, 0 ; 1, \infty)$ as cross ratio, $z \varepsilon C$. The reference numbers come from a cyclic Fibonacci-like sequence. An MT $(\mathrm{z} /(-\mathrm{z}+2))$ maps first $(0,1, \infty) \rightarrow(0,1,-1)$. The inital values for the sequence are $\mathrm{f}_{0}=1, \mathrm{f}_{1}=0$ and $\left.\mathrm{f}_{\mathrm{n}}=\mathrm{f}_{0} \cos (\mathrm{n} \pi / 2)\right)$, $\mathrm{n}$ natural numbers, as solution adds -1 as value in a 4-cycle. The characteristic polynomial is $z^{3}-z^{2}+z$ with roots $0,\left(-p_{1,2}\right)$, the third roots of unity $p_{j}$. Permuting of the reference points is in nucleons for the color charges of quarks in different positions of the SI rotor. The dynamics of SI is a presentation of this $\mathrm{D}_{3}$ symmetry group of order 6 . It is a factor group of $\mathrm{S}_{4}$, the permutation symmetry of four elements. The $r g b$ graviton whirl tip sits in the lower vertex of a tetrahedron for the nucleon with base the quark triangle with color charges $r, g, b$ added at the vertices. The six cross ratios are listed with their $\mathrm{D}_{3}$ symmetry added: $\mathrm{z}$ id, $1 / \mathrm{z} \sigma_{1}, \mathrm{z} /(\mathrm{z}-$ 1) $\alpha \sigma_{1},(\mathrm{z}-1) / \mathrm{z} \alpha^{2},(1-\mathrm{z}) \alpha^{2} \sigma_{1}, 1 /(1-\mathrm{z}) \alpha$. The factoring of $\mathrm{S}_{4}$ to $\mathrm{D}_{3}$ is done by the normal CPT- Klein subgroup $\mathrm{Z}_{2} \mathrm{XZ} \mathrm{Z}_{2}$. Each factor class has four elements; a color charge, a coordinate, one or two symmeties and an energy as force eigenvector. 


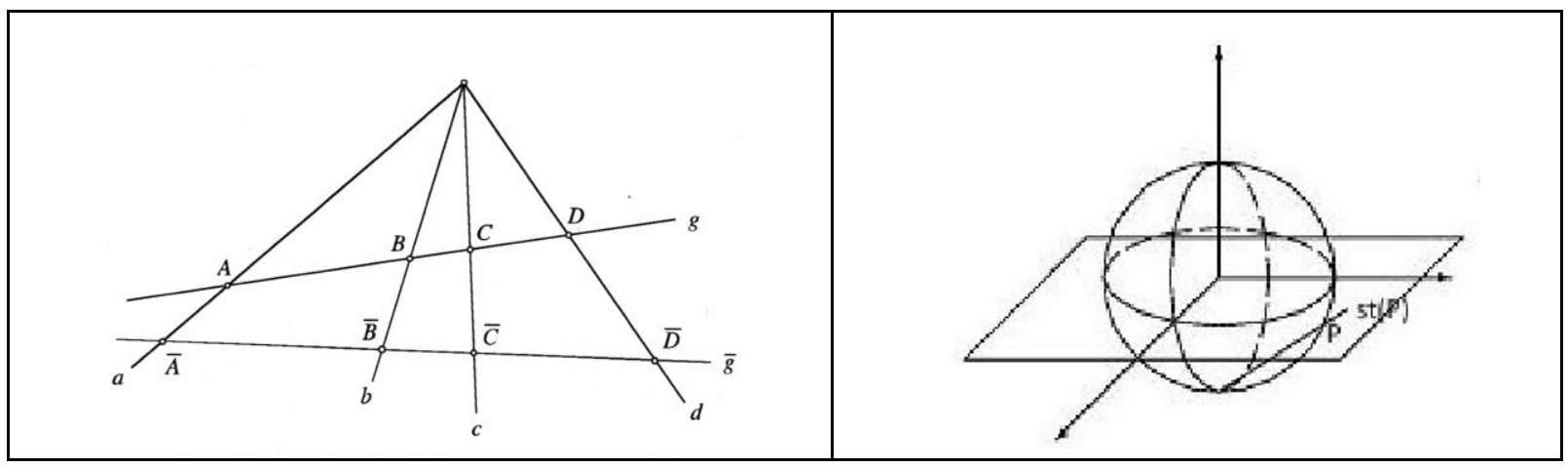

Figure 3 Central projection, a stereographic map

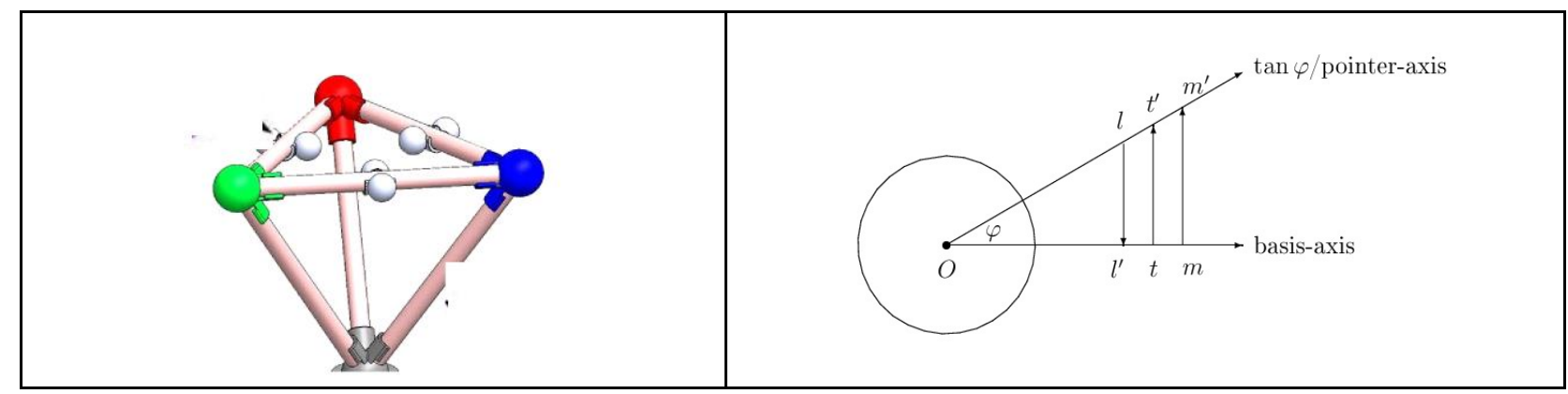

Figure 4 Nucleon with a rgb-graviton GF base triple 126, 3 r-,g-, b-quarks at the upper triangles vertices with gluons ( 2 small balls) for the confining SI energy exchange added on the triangles sides; Minkowski watch for special relativistic norming of force measures with $\cos \varphi$ projections

The numbers (line 7) in the old table below are not for octonians 123456, the last line of natural constants needs revision.

The discrete 6th roots of unity turns of the G-compass needle is generalized. For the leaning angle $\theta$ of E(rot) 3 towards the space $\mathrm{z}$-coordinate, the $r g b$-gavitons use a spiralic orthogonal projection. In the Minkowski watch the sepcial relativistic scaling factor generates a turning angle $\theta$ with $\sin \theta=\mathrm{v} / \mathrm{c}, \mathrm{v}$ relativistic speed and the norming of force measure units by $\cos \theta$ (figure 4 ). A similar angle $\beta$ with $\sin \beta=\mathrm{Rs} / \mathrm{r}$ makes the metrical differentials $\mathrm{dr}$, $\mathrm{dt}$ units $\cos \beta$ rescaling for general relativity [3]. The spiralic repeated orthogonal projections are observed for two galaxies having a common barycenter. They hit after a long time. In discrete form, the orthogonal projections are between two rays with initial point the common barycenter. 
Table 1 Factoring S4 by Z2xZ2 (see [2])

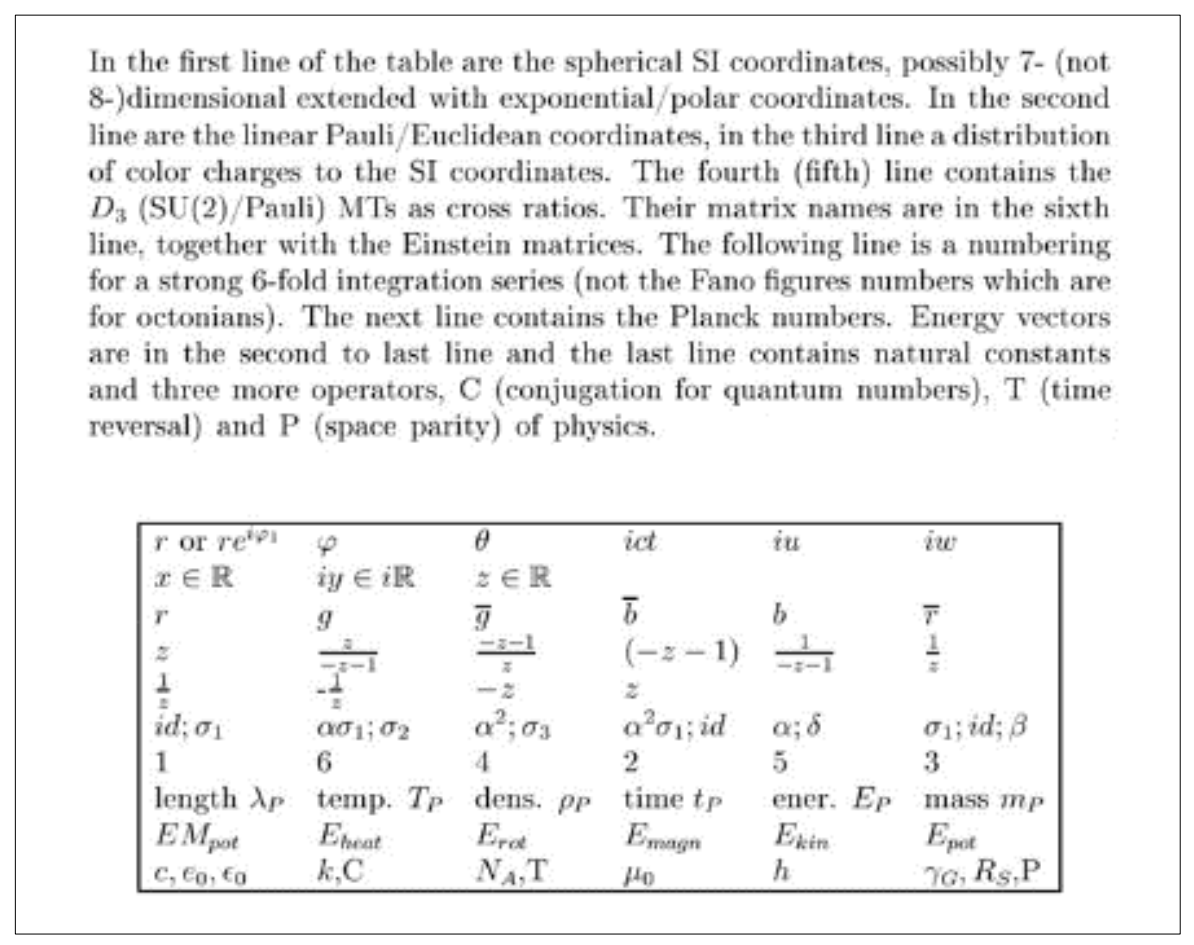

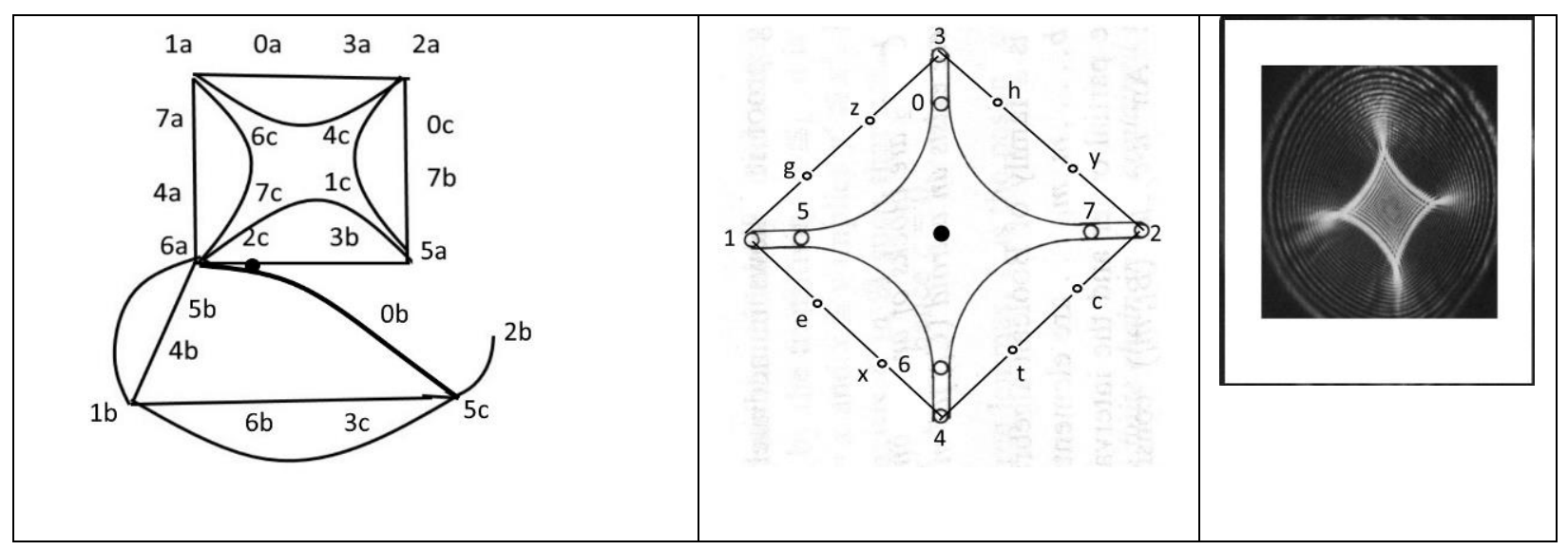

Figure 5 a 4-cycle 1a2a5a6a requires a flash of 4 blocks 1a2a4c6c, 1c2a4c5a, 2c3b5a6a,1a6a6c7c, a 3-cycle 1b5c6a below one block $1 \mathrm{~b} 2 \mathrm{~b} 5 \mathrm{c} 6 \mathrm{c}$ for a 4-dimensional extended $\mathrm{rg} b$-graviton (with time $\mathrm{t}$ added for its wave description $\exp \left(\mathrm{i}(\omega \mathrm{t}+\mathrm{z}), \omega=\mathrm{d} \varphi / \mathrm{dt}\right.$ angular speed); intervals are for blocks $2^{4}$ with 4 atoms as eigen vector lines marked, at superpositions the intervals are broken; an octonian flash: catastrophe (left cusps), at middle 4-cycle 1234 and flash 15 subspace, 03 subspace on top, 27 subspace at right, 46 subspace at bottom (see [1])

Orthogonal projection operators determine through their subspace projections in a 4-dimensional Hilbert space $\mathrm{H}^{4}$ (real or complex) a lattice L [1]. It is the union of its maximal commuting sets of subspaces as blocks $2^{4}$. The blocks are listed by their base vectors of $\mathrm{H}^{4}$. An important observation for the block structure is that 3-and 4-cycles between blocks in superposition require for the lattice structure additional blocks (figure 5). For applications, the required four blocks as flash in the 4-cycle are observed, in the Zeeman gravitational machine (see [5]) as four cusps of four hyperbolic umbilics in superposition. In this view, critical (Morse) functions for manifolds representing potentials are extended to parametric lists of functions $f_{s}$ [5]. Different potential local planes allow jumps when turning the wheels, sudden changes occur as used in decays of energetic unstable systems. There are 1-4 parameters for the available basic seven catastrophes and 1 or 2 variables.

The ellipic umbilic (see [5]) is for the SI rotor. For the weak decays with Heegard decompositions of the 3 dimensional unit sphere $\mathrm{S}^{3}$ as manifold can serve as catastrophe either an umbilic or the butterfly, swallow tail 
catastrophe. For Heegard decompositions of genus $n, n=0,1,2, \ldots$ the number of toroidal handles added to the sphere $\mathrm{S}^{2}$ is due to other dihedral symmetries $\mathrm{D}_{\mathrm{n}}$ than $\mathrm{D}_{3}$ (figure 6).

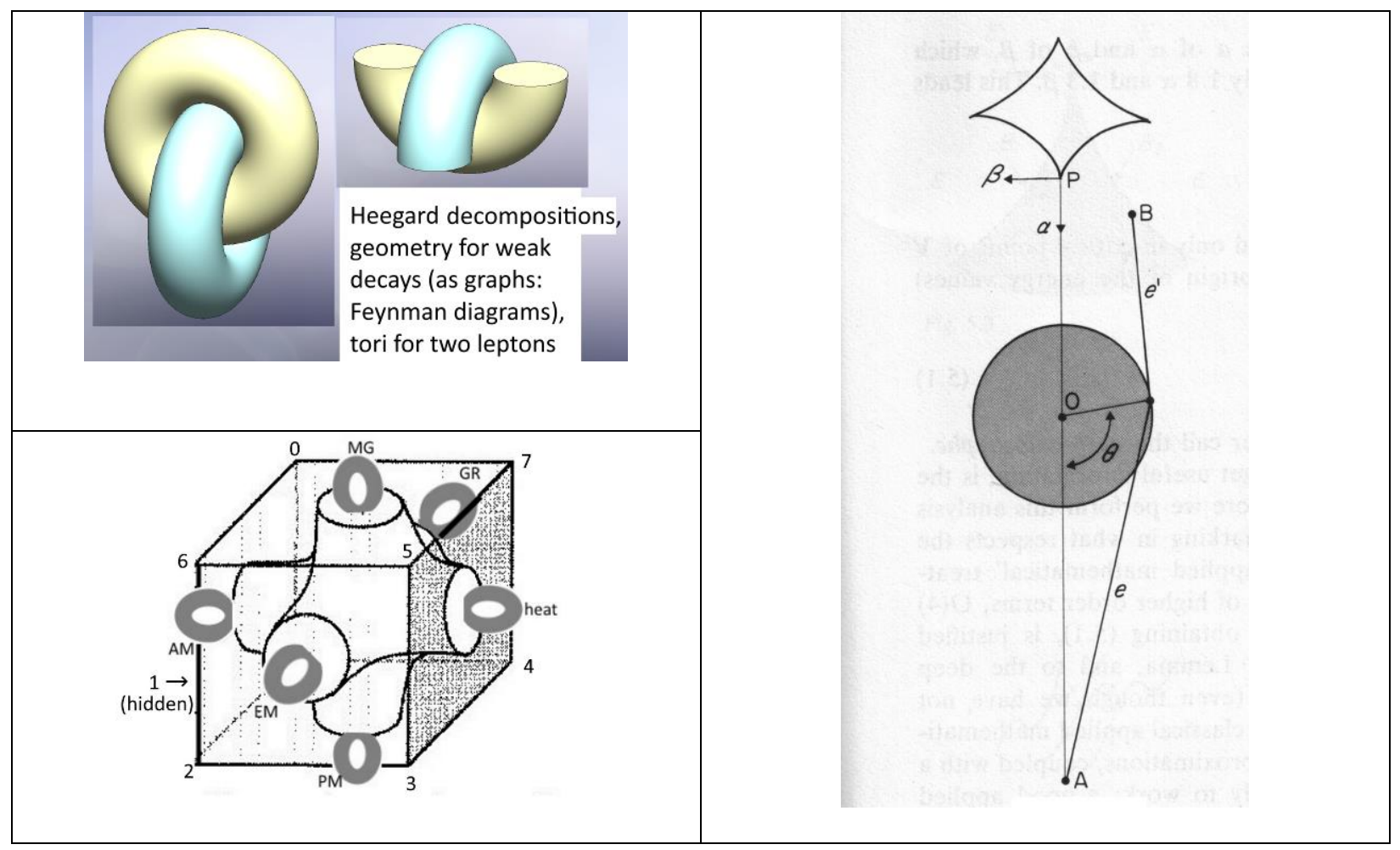

Figure 6 Heeegard decompostion (see [4]) for genus 1 (two tori), below six color charge handles (GR 5, heat 2, EM 1, magn MG 4, PM E(kin) 6 momentum, AM E(rot) 3 angular momentum) for the G-compass added at an octahedrals vertices (replacing a sphere $S^{2}$ ) inside an octonian cube; at right Zeeman machine (see [5])

\section{Big Bang maps}

In the previous sections are mentioned several maps which guide the universes development after a big bang. A black hole is presented as a Horn torus where the radius $r^{\prime}<$ Rs for quarks inside is inverted at the Schwarzschild radius $\mathrm{Rs}$ of the black hole to their universes radius $r$ in $r^{\prime} r=R^{2}$. This mathematical inversion $\mathrm{z} \rightarrow 1 / \mathrm{z}$ at a circle is the first map.

It applies also to dark energy inversions of speeds $v^{\prime}$ inside a pinched torus for dark energy at speed of light in $v^{\prime} v$ $=c^{2}$ for matter (wave) speeds $\mathrm{v}$ in the universe. When SI bifurcates as force from GR, the rgb-graviton length contraction/expansion makes a spiralic pendulum motion for the SI nucleons quark triangle (figure 7).

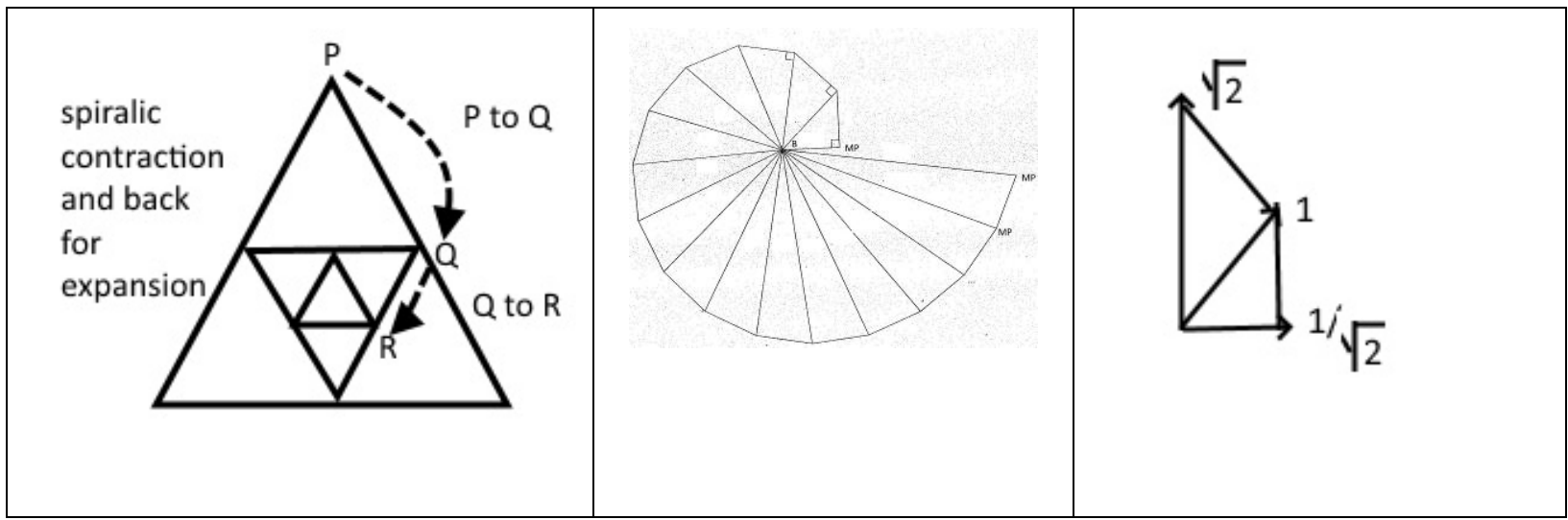

Figure 7 Quark triangles spiralic contraction/expansion, orthogonal spiralic projection (left) for areas in proportion of basic spin lengths $1 / 2: 1: 2$; at middle discrete spiralic rays 
The orthogonal Hilbert space $\mathrm{H}^{4}$ projections onto (closed) subspaces $\mathrm{U}$ gives the decomposition of vectors into two orthogonal vectors; a real vector $x$ splits into two $x=u+w$ with $u \varepsilon U$, we $U^{\perp}$, - the orthogonal complement of $U$ (see [1]). It allows also to extend the real coefficients to complex coefficients of $\mathrm{H}^{4}$. The doubling of spacetime coordinates to octonians is a consequence. The 8 dimensions allow also the 8 gluon GellMann 3x3-matrices as SU(3) symmetry generators. The WI SU(2) Pauli 2x2-matrices are 3x3 extended. They are as quaternion generators for the real $\mathrm{R}^{4}$ spacetime. WI bifurcates from GR and SI. EMI bifurcates later on in the universes development.

The central and stereographic projections of $r g b$-gravitons are for projective spaces. The many projective metrical quadric forms for orbits and shapes of systems arise.

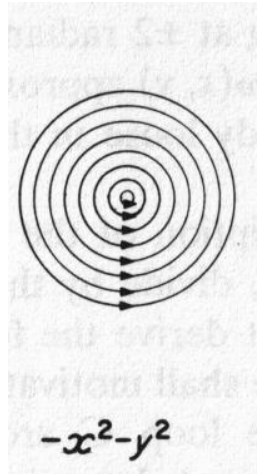

(a)

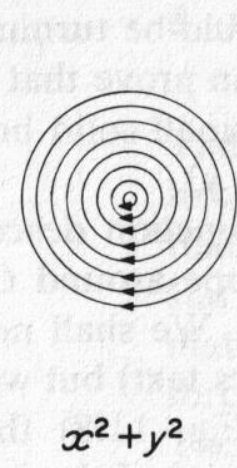

(b)
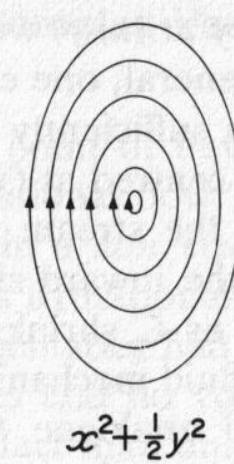

(c)

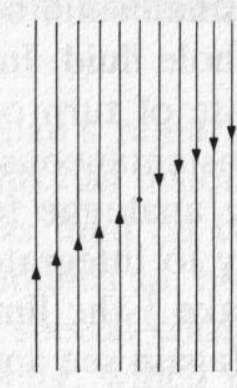

$x^{2}$

(d)

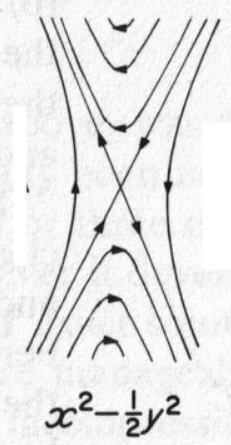

(e)

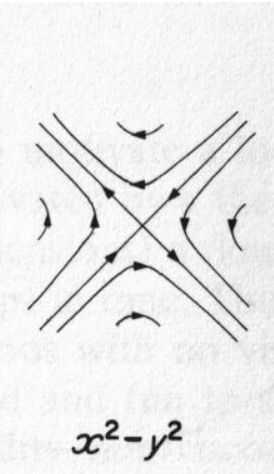

(f)

Figure 8 Quadrics describing flows for potential functions (a)-(c). vector fields (d), quarks 2 roll mill, WI rotor 4 roll mill flow (from [5])

The real and complex projective lines are the U(1) EMI symmetry circle and the Riemannian sphere $S^{2}$. The further projective extensions $\mathrm{P}^{\mathrm{n}}, \mathrm{n} \geq 2$, add to a projective $\mathrm{P}^{(\mathrm{n}-1)}$ space a n-cell $\mathrm{R}^{\mathrm{n}}$ or $\mathrm{C}^{\mathrm{n}}$ to $\mathrm{P}^{\mathrm{n}}=\mathrm{X}^{\mathrm{n}} \mathrm{UP}^{(\mathrm{n}-1)}, \mathrm{X}=\mathrm{R}$ or $\mathrm{C}$, in a topological CW complex extension. The projective duality uses correlations as new symmetries where to a kdimensional subspace an (n-k-1)-subspace of $\mathrm{P}^{\mathrm{n}}$ belongs. To a point (for instance a barycenter with mass scalar attached) corresponds for $\mathrm{n}=2$ a systems (for instance a planet or comet) world line, in rotating about another system Kepler's conic sections (with Einstein's corrrestions added), for $n=3$ surfaces like spheres, a 1 -sheeted hyperbola for leptons, closed at projective infinity to a torus of genus 1, the Minkowski double cone, saddles of the catastrophe umbilics, cylinders for EMI arise. For $n=5$ to a coordinate line g corresponds a 3-dimensional GF as measuring apparatus for this octonian force. A list was given in the first section. Physics adds to the fermionic series a list of 32 quasiparticles to which spin as spinor, rgb-gravitons belong. For E(rot) orbitons can be added, for $\mathrm{E}$ (magn) magnetons, ..., the distribution is not made in this article and needs experimental verifications. Measuring GF's satisfy the Copenhagen interpretation: only one eigenvector is measured as output, the other two remain undetermined and the system can change its state in a measurement. A set of commuting operators can be measured simultaneously. The measures can be extended from real weights to complex or quaternionic weights. The associated Gleason operators give a probability for subspaces where the measured energy (as eigenvector presented) is localized [2].

Remark: Since the GF are more general available, a list of possible extensions to 4-dimensional spaces is added, a eigenvector coordinate, a GF, a 4D-space.

0 color charge force, 037,0237 , color charges, conic whirls G-compass, $S^{2}$ with MT's and cross ratios, Schwarzschild metric

1 EM/WI, 123, 1234, spin R ${ }^{4}$ Hopf fiber bundle, weak bosons, leptons torus of genus 1, real cross product, Minkowski light cone

2 heat, 246, 2467, phonon entropy in volume, pressure

$3 \mathrm{E}(\mathrm{rot}), 347,2347$, orbiton, rotation axis and contour obit

4 E(magn), 145, 1345, cone, induction, magnetic field quantums, magnetic momentum 
5 mass E(pot), 257, 2567, Higgs field and boson, barycenter and barycentrical coordinates

$6 \mathrm{E}$ (kin) momentum $\mathrm{p}=\mathrm{mv}, 356,3456$, SI rotor $\mathrm{CP}^{2}$ and $\mathrm{S}^{5}$ fiber bundle, gluons

7 EMI U(1), 167, 0167, photons, cylinder and unversal cover $R$ of $S^{1}$, quantums $f=n, n=1,2, \ldots$

1256 from above for $r g b$-gravitons 126 and mass 5 is added, projection maps, projective duality and spaces, POT potentials, quarks; the SI projection matrices as 3x3-extension of Pauli matrices generate the projections of $\mathrm{C}^{3}$ 123456 (complex cross product) as 1234, 1256, 3456

Homology and homotopy retracts of 3-dimensional solids obtained with brezel surfaces of genus n (figure 6) are available as Lissajous figures: genus $0 \mathrm{U}(1)$ (EMI) no retract dihedral $\mathrm{D}_{0}$, genus 1 torus (leptons) $\mathrm{S}^{1}$ as dihedral $\mathrm{D}_{1}$ with the conjugation operator for oriented rotations clockwise, counterclockwise (figure 8 (a), (b), (c)), quark brezel genus 2 lemniscate as $\mathrm{D}_{2}$ for POT (electrical, mass/GR potentials) figure 8 (e) and 2 roll mill, nucleon handle body of genus 3 ( 3 quarks) as $\mathrm{D}_{3}$ for the SI rotor presentation (figure 2), 4 roll mill $\mathrm{D}_{4}$ for a weak bosonic inner flow rotation figure 8 (f), $S^{5}$ unit sphere of the SI toplogy for fields R5 as stereographic projection (figure 8 (d)), 6 roll mill $\mathrm{D}_{6}$ as G-compass (figure 2) for color charges and inner nucleon flows; and the Hopf fiber bundle with $S^{3}, S^{2}$ unit spheres and space $R^{3}$ as stereographic $S^{3}$ projection with Heegard decompositions for the genus $n$ solids with orientied 2-dimensionl surfaces of genus $n=0,1,2, \ldots$

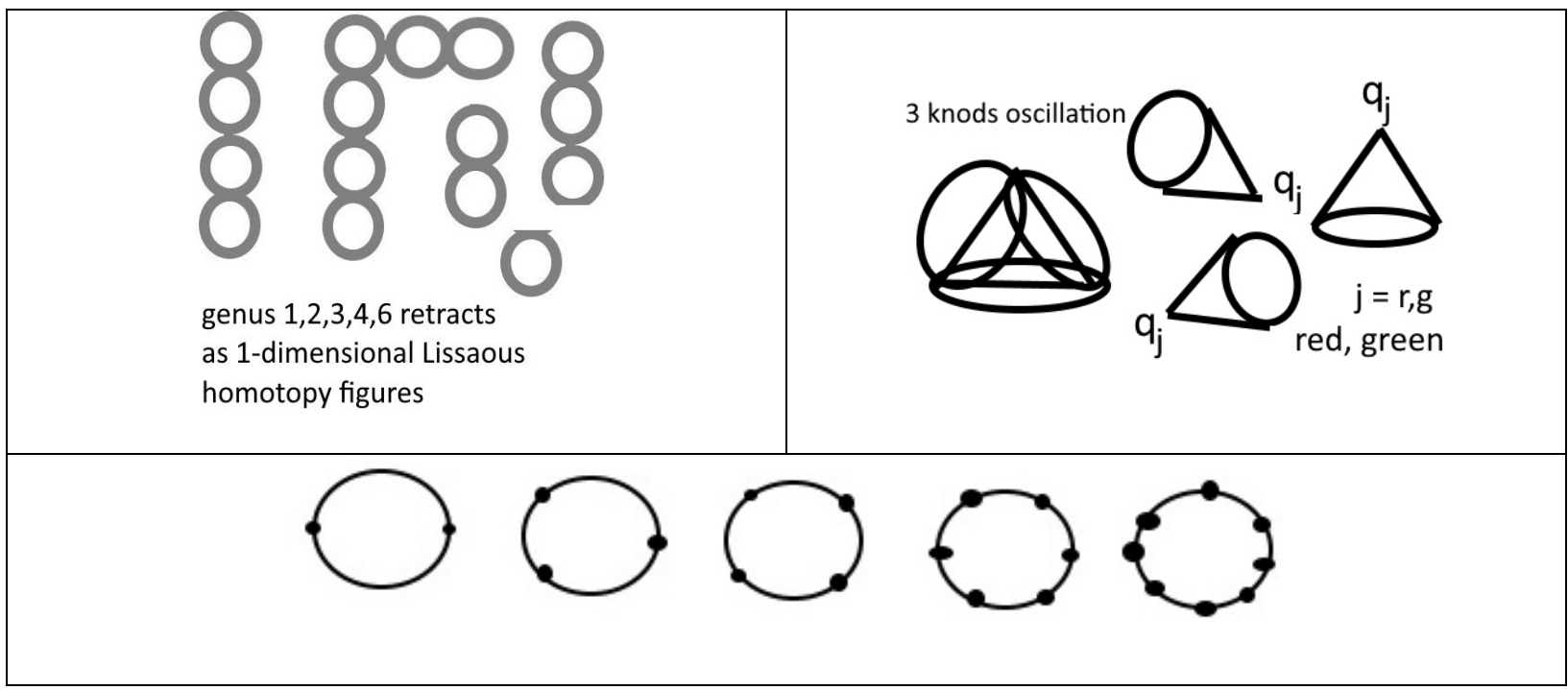

Figure 9 second line dihedrals $D_{n}, n=2,3,4,6,8$, below $D_{3}$ (SI rotor) oscillation; first line 1-dimensional brezel retracts (genus $1,2,3,4,6$ )

$\mathrm{D}_{8}$ is not used as 8 roll mill, as replacement 1234 quaternionic coordinates are bifurcated into octonian 0123456 coordinates; For $\mathrm{D}_{3}$ the roll mill has as elliptic umbilic triangular shaped cusp superpositions (figure 6 at right) and the 3 nucleon quarks are the vertices of a nucleons quark triangle and also the $r g b$-graviton GF as superposition of three color charge whirls contribute to this, an oscillation (figure 9) occurs in form of three cones boundaries with diameter a triangle side where the circles are orthogonal to the triangle surface in space.

\section{Conclusion}

The mathematical description of what happens after a big bang before Planck numbers and physics rules are generated iis modelled according to the Erlanger program (Noether Theorem). The new independent force of color charges belongs with six invariants under Moebius transformations to the complex Riemannian sphere S2. Conserning unit spheres in real or complex spaces, for charges are point locations, S0 iis for dipols are directed vectorial endpoints, S1 as circle is (for the helix lines on an electromagnetic interactions) the transversal cylindrical 
intersecetion and serves as fiber of fiber bundles, S2 is for the color charge force, S3 is the WI Hopf sphere, S5 is the second topological part of the SU(3) geometry S3 x S5 and the space for the nucleon fiber bundle with complex, projective base CP2. The nucleon quark triangle is extended 3-dimensional by the rgb-graviton whirl as in figure 4 left to a tetrahedron configuration. This is responsible for the discrete tetrahedron symmetry group S4 which factors by the CPT Klein group to the quark triangle D3. The factor classes belong to six energies, each having associated a coordinate, a color charge and an eigenvector of a D3 symmetry matrix which gives the measuring unit for the energy. Projective geometry is guiding the model (see [7,8]), Gleason measure operators extend a given energy to spin-like triples as base of S2, dihedrals are used for possible Heegard decompositions of S3. The missing addition to the standard model of physics is provided by new mathematics for gravity and Einstein relativities.

\section{References}

[1] G. Kalmbach. Orthomodular Lattices. - London New York: Academic Press. 1983; 390.

[2] G. Kalmbach H.E. MINT-Wigris. - MINT Verlag Bad Woerishofen. 2019.

[3] G. Kalmbach H.E. Gravity with color charges. J. of Emerging Trends in Engineering and applied Sciences. 2020; 11(5): 183-189.

[4] G. Kalmbach H.E. MINT (Mathematik, Informatik, Naturwissenschaften, Technik), Volume 1-60. Bad Woerishofen: MINT Verlag. 2020.

[5] T. Poston, I. Stewart. Catastrophe theory and its applications, Pitman, London. 1978.

[6] K. Stierstadt, Physik der Materie, VCH, Weinheim. 1989.

[7] MINT-Wigris project (G. Kalmbach H.E.), in the internet under: researchgate.net.

[8] MINT-Wigris, E-Tools, an exhibition of models, located at MINT, Jaudesring 16, D-86825 Bad Woerishofen 